\title{
Experiments of Ultrasonic Sensing Using FBG Sensors
}

\author{
W.W.Shi ${ }^{1, a}$, T.T.Hu ${ }^{1, b}$ and Y.G.Tan ${ }^{1, c}$ \\ ${ }^{1}$ Department of Mechanical and electronic Engineering, Wuhan University of Technology, \\ Wuhan, China \\ ashiww86@126.com, 'bhutingting7002@163.com, cygtan@whut.edu.cn
}

Keywords: Fiber Bragg grating (FBG), Ultrasonic

\begin{abstract}
This research aims at investigating the response characteristics of fiber Bragg grating sensors (FBGs) to ultrasonic signals. The testing system was set up with a tunable laser source and the FBGs installed on the surface of an aluminum plate. Then the response characteristics of FBGs were compared, in condition of putting the ultrasonic driving source in the different longitudinal, lateral and angular separation. Measurements were taken by changing the distance between the sensor and the transducer from $60 \mathrm{~mm}$ to $200 \mathrm{~mm}$ with a step of $20 \mathrm{~mm}$. Then keeping the distance at $100 \mathrm{~mm}$ and $200 \mathrm{~mm}$ respectively, do the angular experiment with the angle from $0^{\circ}$ to $90^{\circ}$ by the step of $10^{\circ}$. Experiment results show that FBG can get better signals when the transducer is along its axial direction. When the location of the transducer is changed linearly, no obvious linear change of the signal strength has been found.
\end{abstract}

\section{Introduction}

Contemporarily, the technology of structural health monitoring has been developing rapidly and modern techniques for non-destructive testing become the focus of attention. Ultrasonic detection, which is one of the traditional methods to detective flaws in the structural, is widely used in damage detections of large flat structures (such as shells of the ship, the wings etc.) as ultrasonic has a prefect directivity. Meanwhile, the emergence of the fiber Bragg grating sensor with its excellent characteristics especially the light quality, essence safety, anti-electromagnetism interference, high sensitivity and can be easily multiplexed has aroused increasing number of researchers get the idea of use both the ultrasonic and FBG sensors to accomplish damage detection. That is employing the FBG sensor, instead of piezoelectric ceramic, to receive ultrasonic signals so that the measuring result can be more accurate. The new approach of combining FBG sensors as well as ultrasonic to detect damages in structures broadens the scope of structural health monitoring (SHM) technique, and to some extent, promotes its development.

The use of FBG sensors to measure ultrasonic has been reported recently. Graham Wild [1] gave a comprehensive and detailed overview in the study of FBGs for ultrasonic measurements and put forward several different experiment methods then some universal conclusions were summarized. Zhang Haiyan [2] set up a testing system in order to detect Lamb wave in aluminum plate by FBG and had investigated the spectral response of FBG subjected to the ultrasonic filed theoretically and numerically. Fomitchov [3] proposed the use of FBG to detect ultrasonic waves in fluid and solid structures. The author used a tunable laser to obtain light signals, which be transformed into electrical signals by photoelectric switches and be measured by photodetector. They found that the response of the sensor is defined by a combined action of direct ultrasonic waves propagating through the medium and axially guided acoustic waves. Hirishi Tsuda $[4,5,6]$ selected several different material plates as experimental samples and researched deeply on damage detection by using FBG to measure ultrasonic waves in various kinds of samples. The related researches are also reported $[7,8,9,10]$. Graham [11] studied the response characteristics of the FBG sensor when the PZT transducer, which was used to generate ultrasonic, was put in the different space positions of aluminum panels. FBG sensor was set in the middle of the panels and measurements were taken for distances of less then 100 $\mathrm{mm}$ and at angles from $0^{\circ}$ to $90^{\circ}$ between the sensor and the transducer. They found that there was no siganificant relation between the location of the PZT transducer and the signal strength recering by FBG sensor. 
The reported literatures show that most of studies focus on how to detect the damages on the basis of using FBG to measure ultrasonic waves that propagating in the differen kinds of structures. However, only a few literatures reported the response characteristics of FBG to ultrasonic waves in metal materials. That is the experimental study in response characteristics of FBG to ultrasonic, especially when the ultrasoic wave is propagating in the metal structure, is not investigated deeply. Therefore, experiments were set up in this paper in order to explore the rules of the intensity of signals obtained by FBG when the PZT transducer was located in different positions which laid the foundation of the development of technology in damage detection basised on FBG-ultrasoic sensor.

\section{Experiments}

The chosen setup for the ultrasonic detection unit can be constructed with the use of tunable laser source, as shown in the Fig. 1 . The size of the aluminum plate is $300 \mathrm{~mm} \times 300 \mathrm{~mm} \times 3 \mathrm{~mm}$. The testing system is consisted by two parts: one is fiber grating sensor system, which used to perceive ultrasonic wave generated by PZT transducer; the other part is ultrasonic generation used to produce ultrasonic. For part one, the tunable laser source is provided by Santec with the model of TSL-210 and the tunable range is $1270-1350 \mathrm{~nm}$. In the experiment, the output power of the laser source was set to $10 \mathrm{~mW}$ and the laser emission wavelength was set to $1290.982 \mathrm{~nm}$. The laser lighter is launched into the FBG sensor via an optical circulator. The FBG, with the center wavelength of $1290.886 \mathrm{~nm}$ and the grating length of $3 \mathrm{~mm}$, was bonded on the surface of the panel. The light reflected from the grating is transmitted into digital oscilloscopes via the photoelectric detector which was provided by Thorlabs with model of PDA10CS-EC and the magnification was set to $3 \mathrm{~dB}$.

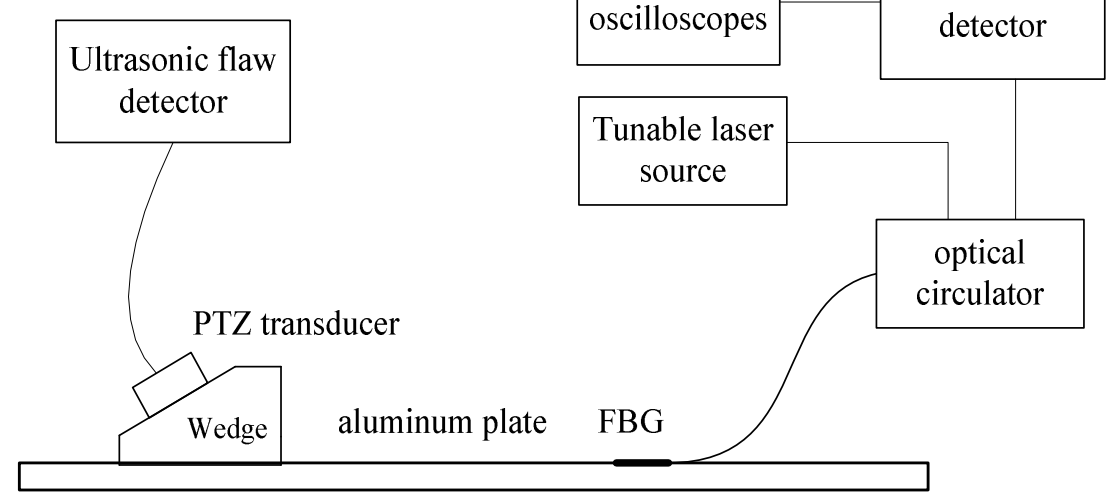

Fig. 1. Schematic diagram of ultrasonic detection unit with a tunable laser source

For the second part, which was used to generate ultrasonic, was showed in Fig.2 and an ultrasonic flaw detector (UFD-Z6) was employed as the source of ultrasonic wave. Parameters were set to the flaw detector: the frequency of the ultrasonic wave was $1 \mathrm{MHz}$ generated by high-energy pulse signal, and set up sound velocity for $6300 \mathrm{~m} / \mathrm{s}$. The transducer is a cylindrical normal probe with the diameter of $20 \mathrm{~mm}$ which can produce longitudinal wave. Put the transducer on the $30^{\circ}$ slope of a wedge which was made from Perspex so that the ultrasonic wave can be transmitted into the panel with the angle of $30^{\circ}$. As shown in the Fig.2, both the wedge and the transducer were placed on the surface of the panel. Acoustic couplant was needed to couple up the surface of the specimen with the wedge; meanwhile the head of the transducer was also coupled up with the slope of a wedge with the same couplant.

A coordinate system was established on the surface of the aluminum panel as showed in the Fig.3. FBG was bonded in the position of coordinate origin, making the axial and radial direction of the optical fiber as two coordinate axes. Then angles must be marked between two coordinate axes by step of $10^{\circ}$. The experiment was divided into two groups. The first group was: move the transducer 


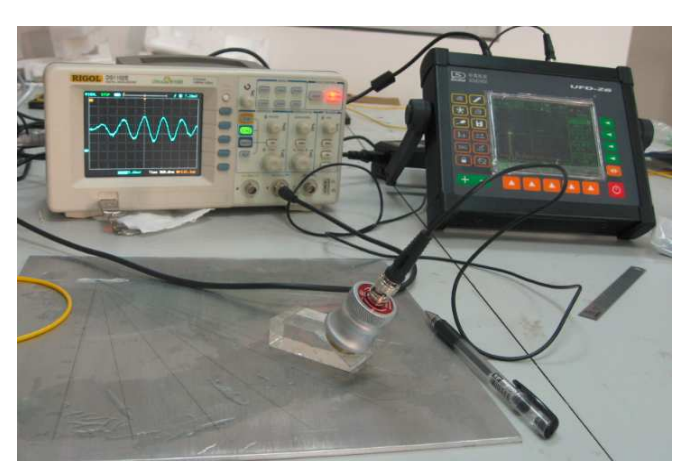

Fig. 2. The way to generate ultrasonic in the experiment

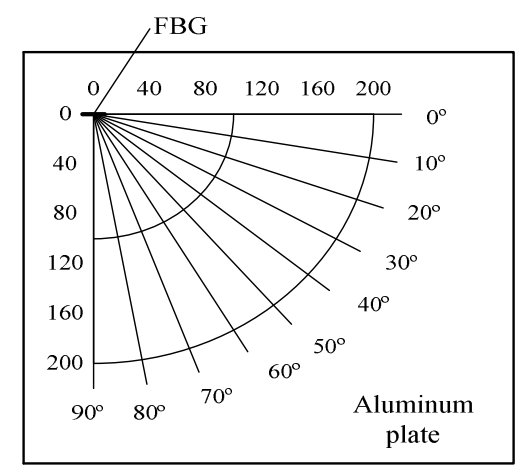

Fig. 3. A coordinate system was established on the surface of the aluminum panel

(and the wedge) along the axial direction of optical fiber. Measurements were taken with a distance of $60 \mathrm{~mm}$ between the FBG and transducer, then increased the distance by step of $20 \mathrm{~mm}$ and recorded the data for 9 times. In the other words, ultrasonic transducer was placed in distance of 60 $\mathrm{mm}, 80 \mathrm{~mm}, 100 \mathrm{~mm} \ldots 200 \mathrm{~mm}$ between FBG respectively and observed changes of waveform which was showed on the oscilloscope, obtain the characteristic signals respectively. It's not uneasy to identify characteristic signals, because a regular sinusoidal signal could be found on the screen of oscilloscope when the ultrasonic was generated. The second group of experiment was: keeping the distance between FBG and transducer constantly for $200 \mathrm{~mm}$, changed the position of the transducer with the angles of $10^{\circ}, 20^{\circ}, 30^{\circ} \ldots 90^{\circ}$ relative to the optical axis and recorded data respectively. Similarly, keeping the distance of $100 \mathrm{~mm}$ constantly and moved transducer angularly got the characteristic signals.

\section{Results}

From the two groups of experiments, the wave shapes of the received characteristic signals by FBG are similar. Fig.4 shows the time domain figure and frequency spectrogram of a characteristic signal. In Fig.4, the centre frequency of the characteristic signal is about $1.12 \mathrm{MHz}$, which explains the received signal is generated by ultrasound. As for the first group of experiment, nine groups of data are collected while transducer is moved in the axial direction of the fiber. Fig. 5 shows the relationship between distance and the intensity of testing signal. As for the experiment of second group, the relationship between angle and the intensity of received signal was showed in Fig.6 which respectively describes the results of the received signals' amplitude with the angle transforming when the distance between driving source and FBG sensor is $200 \mathrm{~mm}$ and $100 \mathrm{~mm}$. When the distance between FBG and the transducer is $100 \mathrm{~mm}$ with the angle shifts to $80^{\circ}$ the received signal has not been recoded in Fig. 6 due to the characteristic signal is difficult to capture.

From the result of the experiment in first group, when the ultrasound propagates in the axial of the fiber, the amplitude of the received signal reduces gradually with the driving source approaches to FBG sensor. However, when the distance between FBG sensor and driving source is $100 \mathrm{~mm}$, the signal increases sharply, and then the signal amplitude increases with the driving source approaches to FBG sensor. Overall, in the axial direction of fiber, the change of the received signal amplitude is not linear with the change of driving source's position. As for the experiment of second group, a gradually decreasing trend has been found to the amplitude of the received signal while the angle approaches to $90^{\circ}$ with the distance is $200 \mathrm{~mm}$. When the distance is $100 \mathrm{~mm}$, the change rule of the amplitude is different, compared with the experiment of $200 \mathrm{~mm}$ distance. There has no linear relation between signal amplitude of the signal and spatial location of driving source which maybe related to the propagating path and style of ultrasound in plate and it will need to be studied in the near future. 

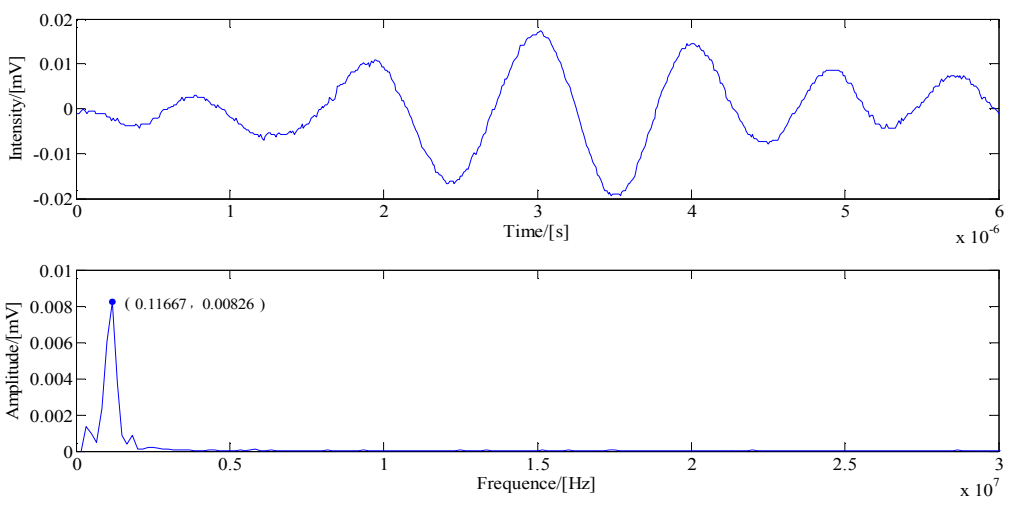

Fig. 4. The T/F domain analysis of characteristic signal

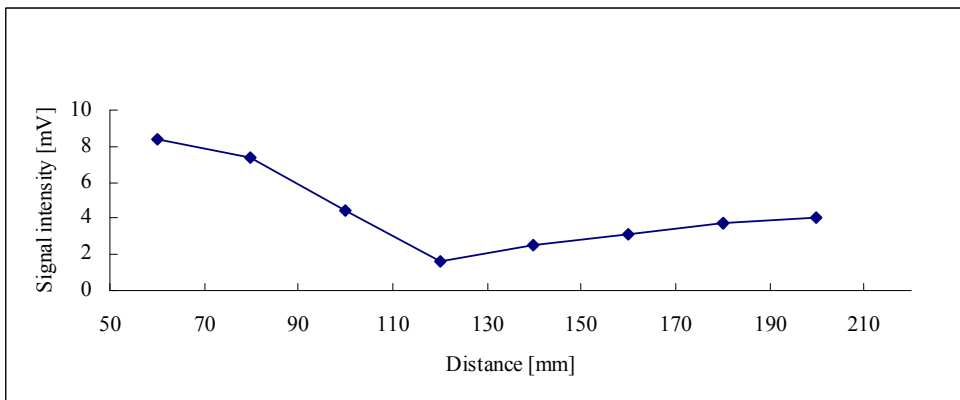

Fig. 5. The relationship between distance and signal intensity

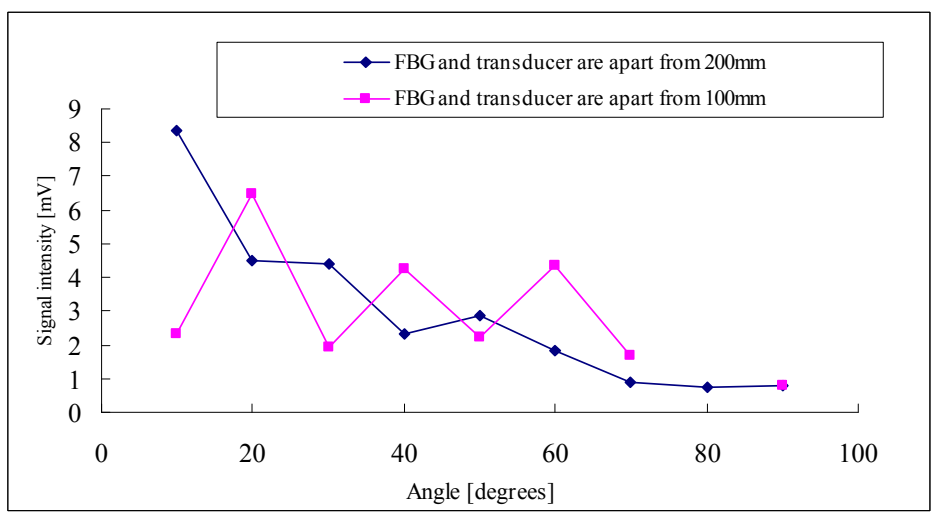

Fig. 6. The relationship between angle and signal intensity

Even so, from the testing data and experimental process, it is obvious that the signal is easy to gain when the driving source is in the axial direction of FBG, the testing amplitude in this direction is higher than the radial direction of the fiber. The two groups of the received signal amplitude are averaged separately. Table 1 shows the signal amplitude is much bigger and easier to obtain when the driving source is put in the axial direction of the optical fiber.

Table 1. The mean value of the two groups of experiments

\begin{tabular}{|c|c|c|}
\hline \multirow{2}{*}{$\begin{array}{c}\text { Distance experiment: the mean } \\
\text { value of signal's intensity [mV] }\end{array}$} & \multicolumn{2}{|c|}{ Angle experiment: the mean value of signal's intensity [mV] } \\
\cline { 2 - 3 } & $\begin{array}{c}\text { FBG and transducer are apart } \\
\text { from 100mm }\end{array}$ & $\begin{array}{c}\text { FBG and transducer are apart from } \\
200 \mathrm{~mm}\end{array}$ \\
\hline 4.399 & 3.004 & 2.972 \\
\hline
\end{tabular}

In this experiment, a tunable laser source is used to demodulate signal, which means the process of collecting characteristic signal is cockamamie, and the stability of the demodulation system decreases obviously when the experimental equipments work long time, which leads to generate noise signal that frequency is $0.2 \mathrm{MHz}$ and the amplitude is $12 \mathrm{mV}$. Thus, effective noise reduction and characteristic signal collection become problems that need to work out in future. 


\section{Conclusion}

This paper investigates the characteristics of FBG's perception to ultrasound by changing the spatial location of the ultrasonic driving source. In the experiments, it's capable for the tunable laser source to test the ultrasound by FBG; when driving source is located in the axial direction of the fiber, it is easy to gain signals and the FBG's sensing effect is much better, while driving source is located in the radial direction of fiber, the FBG's sensing effect is poor; as the location of driving source shifts linearly, the signal amplitude does not show the linear change accordingly.

\section{Acknowledgments}

This project was supported by The National Natural Science Funds (No.51075313).

\section{References}

[1] Graham Wild, Acousto-Ultrasonic Optical Fiber Sensors: Overview and State-of-the-Art, J. IEEE SENSORS JOURNAL. 2008, 8(7):1184-1193

[2] Zhang Haiyan, Ultrasonic Lamb Wave Inspection Using Fiber Bragg Gratings, J. Microwave Conference. 2008,10(12): 805-808

[3] Pavel Fomitchov, Response of a fiber Bragg grating ultrasonic sensor, J. Optical Engineering. 2003, 42(4):956-963

[4] Hiroshi Tsuda, Ultrasonic and damage detection in CFRP using fiber Bragg grating sensors, J. Composites Science and Technology. 2006,(66):676-683

[5] Hiroshi Tsuda, Jung-Ryul Lee, Investigation of fatigue crack in stainless steel using a mobile fiber Bragg grating ultrasonic sensor, J. Optical Fiber Technology. 2007,(13):209-214

[6] Jung-Ryul Lee, Hiroshi Tsuda, Impact wave and damage detection using a strain-free fiber Bragg grating ultrasonic receiver, J. NDT\&E International,2007,(40):85-93

[7] Pou-Man Lan, Acousto-ultrasonic sensing for delaminated GFRP composites using an embedded FBG sensor, J. Optics and Laser in Engineering. 2009,(47):1049-1055

[8] Nobuuo Takeda, Yoji Okabe, Development of smart composite structures with small-diameter fiber Bragg grating sensors for damage detection: Quantitative evaluation of delamination length in CFRP laminates using Lamb wave sensing, J. Composites Science and Technology. 2005,(65):2575-2587

[9] Jung-Ryul Lee, A novel fiber Bragg grating acoustic emission sensor head for mechanical tests, J. Scripta Materialia. 2005,(53):1181-1186

[10] Toshimichi Ogisu, Feasibility studies on active damage detection for CFRP aircraft bonding structures, J. Advanced Composite Materials. 2006, 15(2):153-173

[11] Graham Wild, Spatial Performance of Acousto-Ultrasonic Fiber Bragg Grating Sensor, J. IEEE Sensors Journal. 2010, 10(4):805-806 